\title{
Knowledge, attitude and practice regarding standard precautions among nurses in Teaching Hospital Batticaloa
}

\author{
SGLPN Samarasinghe ${ }^{1}$, RMCD Maithreepala ${ }^{1}$, WN Rangana ${ }^{1}$, MTM Thanis ${ }^{1}$, J Lohitharajah ${ }^{2}$
}

\section{Introduction}

Hospital Acquired infection (HAI) is a major health problem globally and standard precautions are recommended to prevent HAI. As part of the health care team, nurses play a vital role in controlling HAI. This study was aimed to assess the knowledge, attitude and practice (KAP) of nurses about standard precautions (SP).

\section{Methods}

A descriptive cross-sectional study was conducted among nurses working in Teaching Hospital Batticaloa between February-March 2020. A pretested, structured self-administered questionnaire assessing core elements of KAP on standard precautions was used to collect the data. Percentage KAP scores were calculated and the level of KAP were determined. Descriptive analysis was done using SPSS software v.25.

\section{Results}

A total of 367 nurses participated in the study of whom $41.1 \%$ and $35.4 \%$ were in the age groups of 21-30 and 31-40 years respectively with male: female ratio of 1:3.8. Most of the participants $(42.5 \%)$ were working in wards with experience ranging from 01 month to 38 years. Only $13.4 \%$ received training on SP while 3.3\% of participants were unaware about SP. Most of the participants $(53.1 \%)$ had heard of SP mainly from the infection control unit while $21.8 \%$ learned from their own experience. Among the participants $73.8 \%$ had good knowledge, $56.4 \%$ had an average attitude and $94.6 \%$ had good practice. Knowledge score was significantly associated with place of work $(\mathrm{p}=0.018)$ and work experience $(\mathrm{p}=0.007)$.

\section{Conclusion}

Educational programs concerning various aspects of standard precautions is recommended to strengthen knowledge, alter attitude and enhance adherence of nursing professionals to SPs.

Keywords: Standard precaution (SP), knowledge, attitude, practice, Nurses

\footnotetext{
${ }^{1}$ Department of Supplementary Health Sciences, Faculty of Health-Care Sciences, Eastern University, Sri Lanka ${ }^{2}$ Department of Pathophysiology, Faculty of Health-Care Sciences, Eastern University, Sri Lanka 